\title{
Common coupled fixed point results in multiplicative metric spaces and applications
}

\author{
Yun Jiang, Feng Gu* \\ Institute of Applied Mathematics and Department of Mathematics, Hangzhou Normal University, Hangzhou, Zhejiang 310036, China.
}

Communicated by W. Shatanawi

\begin{abstract}
In the framework of a multiplicative metric space, by using the concept of $w^{*}$-compatible mappings, we establish some new common coupled fixed point theorems for two mappings satisfying $\phi$-type contractive condition. We do not use the condition of continuity of any mapping for finding the coupled coincidence and common coupled fixed point. Meantime, we also provide some examples to support our new results. As an application, we provide an existence and uniqueness theorem of common solution for a class of nonlinear integral equations by using the obtained new result. (c)2017 All rights reserved.
\end{abstract}

Keywords: Multiplicative metric spaces, coupled coincidence point, coupled common fixed point, $w^{*}$-compatible mapping pairs.

2010 MSC: 47H10, 54H25.

\section{Introduction and preliminaries}

In 2008, Bashirov et al. [4] first introduced a new concept of multiplicative metric spaces, discussed some problems of multiplicative calculus, and proved the basic theorem of multiplicative calculus. In 2012, Bashirov et al. [5] demonstrated the efficiency of multiplicative calculus over the Newtonian calculus. They elaborated that multiplicative calculus is more effective than Newtonian calculus for modeling various problems from different fields.

Since the multiplicative metric spaces was introduced, many authors got many fixed point theorems in multiplicative metric spaces, such as, Ozavsar and Cevikel [16] proved some fixed point theorems of multiplicative contraction mappings in multiplicative spaces, He et al. [13] established common fixed point theorems for weak commutative mappings in the setting of multiplicative metric space, and Abbas et al. [1] proved some common fixed point results of quasi-weak commutative mappings on a closed ball in the framework of the multiplicative metric spaces. Very recently, Gu and Cho [9] established common fixed point results for four maps satisfying $\phi$-contractive condition in multiplicative metric spaces.

In 2006, Bhaskar and Lakshmikantham [7] introduced the concept of a coupled fixed point and they used the property of the mixed monotone property to prove some coupled fixed point theorems. Later,

\footnotetext{
*Corresponding author

Email addresses: $1807862306 @ q q$. com (Yun Jiang), gufeng99@sohu.com (Feng Gu)
} 
Lakshmikantham and Ćirić [15] extended and generalized the former result by introducing a new concept of coupled coincidence point of mappings and using the mixed $g$-monotone property. For some works on a coupled fixed point in different spaces, we refer the reader to [2, 3, 6, 8, 10-12, 14, 17-19]. Nevertheless, so far, there is no any author to research the problems of coupled fixed point in multiplicative metric spaces. The purpose of this article is to establish some new common coupled fixed point theorems for two mappings satisfying $\phi$-type contractive condition in multiplicative metric spaces. Furthermore, we also provide some illustrative examples in support of our new results. As an application, we also provide an existence and uniqueness theorem of common solution for a class of nonlinear integral equations by using the obtained new result.

In this section, we first introduce some basic notions and known results.

Definition 1.1 ([4]). Let $X$ be a nonempty set. A multiplicative metric is a mapping $d: X \times X \rightarrow \mathbb{R}^{+}$ satisfying the following axioms

(M1) $d(x, y) \geqslant 1, \forall x, y \in X, d(x, y)=1 \Leftrightarrow x=y$;

(M2) $d(x, y)=d(y, x), \forall x, y \in X$

(M3) $d(x, y) \leqslant d(x, z) \cdot d(z, y), \forall x, y, z \in X$ (multiplicative triangle inequality).

The pair $(X, d)$ is called a multiplicative metric spaces.

Example 1.2 ([4]). Let $\mathbb{R}_{+}^{n}$ be the collection of all $n$-tuples of positive real numbers. Let $d: \mathbb{R}_{+}^{n} \times \mathbb{R}_{+}^{n} \rightarrow \mathbb{R}$ be defined by

$$
\mathrm{d}(x, y)=\left|\frac{x_{1}}{y_{1}}\right| \cdot\left|\frac{x_{2}}{y_{2}}\right| \cdots\left|\frac{x_{n}}{y_{n}}\right|
$$

where $X=\left(x_{1}, \cdots, x_{n}\right), y=\left(y_{1} \cdots, y_{n}\right) \in \mathbb{R}_{+}^{n}$, and $|\cdot|: \mathbb{R}_{+}^{n} \rightarrow \mathbb{R}_{+}^{n}$ is defined by

$$
|a|= \begin{cases}a, & a \geqslant 1 \\ \frac{1}{a}, & a<1\end{cases}
$$

Then it is obvious that all conditions of multiplicative metric are satisfied.

Definition $1.3([4])$. Let $(X, d)$ be a multiplicative metric spaces, $\left\{x_{n}\right\}$ be a sequence in $X$, and $x \in X$. If for every multiplicative open ball $B_{\epsilon}(x)=\{y \mid d(x, y)<\epsilon\}, \epsilon>1$, there exists a natural number $N \in \mathbb{N}$ such that $n \geqslant N$ then $x_{n} \in B_{\epsilon}(x)$. Then the sequence $\left\{x_{n}\right\}$ is said to be multiplicative converging to $x$, denoted by $x_{n} \rightarrow x(n \rightarrow \infty)$.

Proposition $1.4([16])$. Let $(X, d)$ be a multiplicative metric space, $\left\{x_{n}\right\}$ be sequence in $X$ and $x \in X$. Then

$$
x_{n} \rightarrow x(n \rightarrow \infty) \Leftrightarrow d\left(x_{n}, x\right) \rightarrow 1(n \rightarrow \infty) .
$$

Definition $1.5([16])$. Let $(X, d)$ be a multiplicative metric space, and $\left\{x_{n}\right\}$ be a sequence in $X$. The sequence $x \in X$ is called multiplicative Cauchy sequence if, for each $\epsilon>0$, there exists a positive integer $N \in \mathbb{N}$ such that $d\left(x_{n}, x_{m}\right)<\epsilon$ for all $n, m \geqslant N$.

Proposition 1.6 ([16]). Let $(X, d)$ be a multiplicative metric space and $\left\{x_{n}\right\}$ be a sequence in $X$. Then $\left\{x_{n}\right\}$ is a multiplicative Cauchy sequence if and only if $\mathrm{d}\left(\mathrm{x}_{\mathrm{n}}, \mathrm{x}_{\mathrm{m}}\right) \rightarrow 1(\mathrm{n}, \mathrm{m} \rightarrow \infty)$.

Definition 1.7 ([16]). A multiplicative metric space $(X, d)$ is said to be multiplicative complete if every multiplicative Cauchy sequence in $(X, d)$ is multiplicative convergent in $X$.

Definition 1.8. ([16]) Let $\left(X, d_{X}\right)$ and $\left(Y, d_{Y}\right)$ be two multiplicative metric spaces and $f: X \rightarrow Y$ be a function. If for $f$ holds the requirement that, for every $\epsilon>1$, there exists $\delta>1$ such that $f\left(B_{\delta}(x)\right) \subset$ $B_{\epsilon}(f(x))$, then we call $f$ multiplicative continuous at $x \in X$.

Proposition $1.9([16])$. Let $\left(X, \mathrm{~d}_{\mathrm{X}}\right)$ and $\left(\mathrm{Y}, \mathrm{d}_{\mathrm{Y}}\right)$ be two multiplicative metric spaces, $\mathrm{f}: \mathrm{X} \rightarrow \mathrm{Y}$ be a mapping. Then $f$ is multiplicative continuous at $x \in X$ if and only if $f\left(x_{n}\right) \rightarrow f(x)$ for every sequence $\left\{x_{n}\right\} \subset X$ with $x_{n} \rightarrow$ $x(n \rightarrow \infty)$. 
Proposition $1.10([16])$. Let $(X, d)$ be multiplicative metric space, and $\left\{x_{n}\right\}$ and $\left\{y_{n}\right\}$ be two sequences in $X$ such that $x_{n} \rightarrow x, y_{n} \rightarrow y(n \rightarrow \infty), x, y \in X$. Then

$$
d\left(x_{n}, y_{n}\right) \rightarrow d(x, y)(n \rightarrow \infty)
$$

Definition 1.11 ([7]). Let $X$ be a nonempty set. We call an element $(x, y) \in X \times X$ a coupled fixed point of the mapping $F: X \times X \rightarrow X$ if $F(x, y)=x$ and $F(y, x)=y$.

Definition 1.12 ([15]). Let $X$ be a nonempty set. An element $(x, y) \in X \times X$ is called

(i) a coupled coincidence point of the mappings $F: X \times X \rightarrow X$ and $g: X \rightarrow X$ if $F(x, y)=g x$ and $F(y, x)=g y$; in this case $(g x, g y)$ is called coupled point of coincidence of mappings $F$ and $g$;

(ii) a common coupled fixed point of mappings $F: X \times X \rightarrow X$ and $g: X \rightarrow X$ if $F(x, y)=g x=x$ and $F(y, x)=g y=y$.

Definition 1.13 ([2]). Let $X$ be a nonempty set. We say that the mappings $F: X \times X \rightarrow X$ and $g: X \rightarrow X$ are called

(i) $w$-compatible if $g F(x, y)=F(g x, g y)$ whenever $F(x, y)=g x$ and $F(y, x)=g y$;

(ii) $w^{*}$-compatible $g F(x, x)=F(g x, g x)$ whenever $F(x, x)=g x$.

Let $\Phi$ denote the set of functions $\phi:[1, \infty)^{5} \rightarrow[0, \infty)$ satisfying

(1) $\phi$ is nondecreasing and continuous in each coordinate variable;

(2) for $t \geqslant 1$,

$$
\psi(t)=\max \{\phi(t, t, t, 1, t), \phi(t, t, t, t, 1), \phi(t, 1,1, t, t), \phi(1, t, 1, t, 1), \phi(1,1, t, 1, t)\} \leqslant t .
$$

Form now on, unless otherwise stated, we choose $\phi \in \Phi$.

\section{Main results}

Theorem 2.1. Let $(\mathrm{X}, \mathrm{d})$ be a multiplicative metric space, $\mathrm{F}: \mathrm{X} \times \mathrm{X} \rightarrow \mathrm{X}$ and $\mathrm{g}: \mathrm{X} \rightarrow \mathrm{X}$ be two mappings. Suppose that there exists $\lambda \in(0,1)$ such that the condition

$$
d(F(x, y), F(u, v)) \cdot d(F(y, x), F(v, u)) \leqslant \phi\left(\begin{array}{c}
d^{\lambda}(g x, g u) \cdot d^{\lambda}(g y, g v), \\
d^{\lambda}(F(x, y), g x) \cdot d^{\lambda}(F(y, x), g y), \\
d^{\lambda}(F(u, v), g u) \cdot d^{\lambda}(F(v, u), g v), \\
d^{\lambda}(F(x, y), g u) \cdot d^{\lambda}(F(y, x), g v), \\
d^{\lambda}(F(u, v), g x) \cdot d^{\lambda}(F(v, u), g y)
\end{array}\right)
$$

holds for all $(\mathrm{x}, \mathrm{y}),(\mathrm{u}, v) \in \mathrm{X} \times \mathrm{X}$. If $\mathrm{F}(\mathrm{X} \times \mathrm{X}) \subset \mathrm{g}(\mathrm{X}), \mathrm{g}(\mathrm{X})$ is a multiplicative complete subspace of $\mathrm{X}$, and $\mathrm{F}$ and $\mathrm{g}$ are $\omega^{*}$-compatible, then $\mathrm{F}$ and $\mathrm{g}$ have a unique common coupled fixed point of the form $(\mathrm{u}, \mathrm{u}) \in \mathrm{X} \times \mathrm{X}$.

Proof. Let $x_{0}, y_{0} \in X$. Since $F(X \times X) \subset g(X)$, we can choose $x_{1}, y_{1} \in X$ such that $g x_{1}=F\left(x_{0}, y_{0}\right)$ and $g y_{1}=F\left(y_{0}, x_{0}\right)$. Similarly, we can choose $\left(x_{2}, y_{2}\right) \in X$ such that $g x_{2}=F\left(x_{1}, y_{1}\right)$ and $g y_{2}=F\left(y_{1}, x_{1}\right)$. Continuing in this way, then we can construct two sequences $\left\{x_{n}\right\}$ and $\left\{y_{n}\right\}$ in $X$, such that

$$
g x_{n+1}=F\left(x_{n}, y_{n}\right), \quad g y_{n+1}=F\left(y_{n}, x_{n}\right), \quad \forall n \geqslant 0 .
$$

If follows from (2.1), (2.2), (M3), and function $\phi$, and also the property of $\psi$ that

$$
\begin{aligned}
& d\left(g x_{n}, g x_{n+1}\right) \cdot d\left(g y_{n}, g y_{n+1}\right) \\
& =d\left(F\left(x_{n-1}, y_{n-1}\right), F\left(x_{n}, y_{n}\right)\right) \cdot d\left(F\left(y_{n-1}, x_{n-1}\right), F\left(y_{n}, x_{n}\right)\right)
\end{aligned}
$$




$$
\begin{aligned}
& \leqslant \phi\left(\begin{array}{c}
d^{\lambda}\left(g x_{n-1}, g x_{n}\right) \cdot d^{\lambda}\left(g y_{n-1}, g y_{n}\right), \\
d^{\lambda}\left(F\left(x_{n-1}, y_{n-1}\right), g x_{n-1}\right) \cdot d^{\lambda}\left(F\left(y_{n-1}, x_{n-1}\right), g y_{n-1}\right) \\
d^{\lambda}\left(F\left(x_{n}, y_{n}\right), g x_{n}\right) \cdot d^{\lambda}\left(F\left(y_{n}, x_{n}\right), g y_{n}\right), \\
d^{\lambda}\left(F\left(x_{n-1}, y_{n-1}\right), g x_{n}\right) \cdot d^{\lambda}\left(F\left(y_{n-1}, x_{n-1}\right), g y_{n}\right) \\
d^{\lambda}\left(F\left(x_{n}, y_{n}\right), g x_{n-1}\right) \cdot d^{\lambda}\left(F\left(y_{n}, x_{n}\right), g y_{n-1}\right)
\end{array}\right) \\
& =\phi\left(\begin{array}{c}
d^{\lambda}\left(g x_{n-1}, g x_{n}\right) \cdot d^{\lambda}\left(g y_{n-1}, g y_{n}\right), \\
d^{\lambda}\left(g x_{n}, g x_{n-1}\right) \cdot d^{\lambda}\left(g y_{n}, g y_{n-1}\right), \\
d^{\lambda}\left(g x_{n+1}, g x_{n}\right) \cdot d^{\lambda}\left(g y_{n+1}, g y_{n}\right), \\
d^{\lambda}\left(g x_{n}, g x_{n}\right) \cdot d^{\lambda}\left(g y_{n}, g y_{n}\right), \\
d^{\lambda}\left(g x_{n+1}, g x_{n-1}\right) \cdot d^{\lambda}\left(g y_{n+1}, g y_{n-1}\right)
\end{array}\right) \\
& \leqslant \phi\left(\begin{array}{c}
d^{\lambda}\left(g x_{n-1}, g x_{n}\right) \cdot d^{\lambda}\left(g y_{n-1}, g y_{n}\right), d^{\lambda}\left(g x_{n}, g x_{n-1}\right) \cdot d^{\lambda}\left(g y_{n}, g y_{n-1}\right), \\
d^{\lambda}\left(g x_{n+1}, g x_{n}\right) \cdot d^{\lambda}\left(g y_{n+1}, g y_{n}\right), 1 \\
d^{\lambda}\left(g x_{n+1}, g x_{n}\right) \cdot d^{\lambda}\left(g x_{n}, g x_{n-1}\right) \cdot d^{\lambda}\left(g y_{n+1}, g y_{n}\right) \cdot d^{\lambda}\left(g y_{n}, g y_{n-1}\right)
\end{array}\right) \\
& \leqslant \phi\left(\begin{array}{c}
d^{\lambda}\left(g x_{n-1}, g x_{n}\right) \cdot d^{\lambda}\left(g y_{n-1}, g y_{n}\right) \cdot d^{\lambda}\left(g x_{n}, g x_{n+1}\right) \cdot d^{\lambda}\left(g y_{n}, g y_{n+1}\right), \\
d^{\lambda}\left(g x_{n-1}, g x_{n}\right) \cdot d^{\lambda}\left(g y_{n-1}, g y_{n}\right) \cdot d^{\lambda}\left(g x_{n}, g x_{n+1}\right) \cdot d^{\lambda}\left(g y_{n}, g y_{n+1}\right), \\
d^{\lambda}\left(g x_{n-1}, g x_{n}\right) \cdot d^{\lambda}\left(g y_{n-1}, g y_{n}\right) \cdot d^{\lambda}\left(g x_{n}, g x_{n+1}\right) \cdot d^{\lambda}\left(g y_{n}, g y_{n+1}\right), 1, \\
d^{\lambda}\left(g x_{n-1}, g x_{n}\right) \cdot d^{\lambda}\left(g y_{n-1}, g y_{n}\right) \cdot d^{\lambda}\left(g x_{n}, g x_{n+1}\right) \cdot d^{\lambda}\left(g y_{n}, g y_{n+1}\right)
\end{array}\right) \\
& \leqslant \psi\left(d^{\lambda}\left(g x_{n-1}, g x_{n}\right) \cdot d^{\lambda}\left(g y_{n-1}, g y_{n}\right) \cdot d^{\lambda}\left(g x_{n}, g x_{n+1}\right) \cdot d^{\lambda}\left(g y_{n}, g y_{n+1}\right)\right) \\
& \leqslant d^{\lambda}\left(g x_{n-1}, g x_{n}\right) \cdot d^{\lambda}\left(g y_{n-1}, g y_{n}\right) \cdot d^{\lambda}\left(g x_{n}, g x_{n+1}\right) \cdot d^{\lambda}\left(g y_{n}, g y_{n+1}\right) \text {, }
\end{aligned}
$$

which implies that

$$
\begin{aligned}
& d\left(g x_{n}, g x_{n+1}\right) \cdot d\left(g y_{n}, g y_{n+1}\right) \\
& \quad \leqslant d^{\frac{\lambda}{1-\lambda}}\left(g x_{n-1}, g x_{n}\right) \cdot d^{\frac{\lambda}{1-\lambda}}\left(g y_{n-1}, g y_{n}\right)=d^{h}\left(g x_{n-1}, g x_{n}\right) \cdot d^{h}\left(g y_{n-1}, g y_{n}\right) .
\end{aligned}
$$

Here $h=\frac{\lambda}{1-\lambda} \in(0,1)$. It follows from (2.3) that

$$
\begin{aligned}
d\left(g x_{n}, g x_{n+1}\right) \cdot d\left(g y_{n}, g y_{n+1}\right) & \leqslant d^{h}\left(g x_{n-1}, g x_{n}\right) \cdot d^{h}\left(g y_{n-1}, g y_{n}\right) \\
& \leqslant d^{h^{2}}\left(g x_{n-2}, g x_{n-1}\right) \cdot d^{h^{2}}\left(g y_{n-2}, g y_{n-1}\right) \\
& \vdots \\
& \leqslant d^{h^{n}}\left(g x_{0}, g x_{1}\right) \cdot d^{h^{n}}\left(g y_{0}, g y_{1}\right) .
\end{aligned}
$$

Therefore, for all $n \in \mathbb{N}, n<m$, by the multiplicative triangle inequality we obtain

$$
\begin{aligned}
& d\left(g x_{n}, g x_{m}\right) \cdot d\left(g y_{n}, g y_{m}\right) \\
& \leqslant d\left(g x_{n}, g x_{n+1}\right) \cdot d\left(g x_{n+1}, g x_{n+2}\right) \cdots d\left(x_{m-1}, x_{m}\right) \cdot d\left(g y_{n}, g y_{n+1}\right) \\
& \quad \cdot d\left(g y_{n+1}, g y_{n+2}\right) \cdots d\left(g y_{m-1}, g y_{m}\right) \\
& \leqslant d^{h^{n}}\left(g x_{0}, g x_{1}\right) \cdot d^{h^{n}}\left(g y_{0}, g y_{1}\right) \cdot d^{h^{n+1}}\left(g x_{0}, g x_{1}\right) \cdot d^{h^{n+1}}\left(g y_{0}, g y_{1}\right) \cdots d^{h^{m-1}}\left(g x_{0}, g x_{1}\right) \\
& \quad \cdot d^{h^{m-1}}\left(g y_{0}, g y_{1}\right) \\
& \leqslant d^{\frac{h^{n}}{1-h}}\left(g x_{0}, g x_{1}\right) \cdot d^{h^{n}}\left(g y_{0}, g y_{1}\right) .
\end{aligned}
$$

This implies that

$$
d\left(g x_{n}, g x_{m}\right) \cdot d\left(g y_{n}, g y_{m}\right) \rightarrow 1(n, m \rightarrow \infty)
$$

such that

$$
d\left(g x_{n}, g x_{m}\right) \rightarrow 1(n, m \rightarrow \infty), \quad d\left(g y_{n}, g y_{m}\right) \rightarrow 1(n, m \rightarrow \infty)
$$


Hence $\left\{g x_{n}\right\}$ and $\left\{g y_{n}\right\}$ are Cauchy sequences in $g(X)$. By completeness of $g(X)$, there exist $g x, g y \in g(X)$ such that $\left\{g x_{n}\right\}$ and $\left\{g y_{n}\right\}$ converge to $g x$ and $g y$, respectively. Now we prove that $F(x, y)=g x$ and $F(y, x)=g y$. In fact, it follows from (M3) and (2.1) that

$$
\begin{aligned}
& d(F(x, y), g x) \cdot d(F(y, x), g y) \\
& \leqslant d\left(F(x, y), g x_{n+1}\right) \cdot d\left(g x_{n+1}, g x\right) \cdot d\left(F(y, x), g y_{n+1}\right) \cdot d\left(g y_{n+1}, g y\right) \\
& =d\left(F(x, y), F\left(x_{n}, y_{n}\right)\right) \cdot d\left(F(y, x), F\left(y_{n}, x_{n}\right)\right) \cdot d\left(g x_{n+1}, g x\right) \cdot d\left(g y_{n+1}, g y\right) \\
& \leqslant \phi\left(\begin{array}{c}
d^{\lambda}\left(g x, g x_{n}\right) \cdot d^{\lambda}\left(g y, g y_{n}\right), \\
d^{\lambda}(F(x, y), g x) \cdot d^{\lambda}(F(y, x), g y), \\
d^{\lambda}\left(F\left(x_{n}, y_{n}\right), g x_{n}\right) \cdot d^{\lambda}\left(F\left(y_{n}, x_{n}\right), g y_{n}\right), \\
d^{\lambda}\left(F(x, y), g x_{n}\right) \cdot d^{\lambda}\left(F(y, x), g y_{n}\right), \\
d^{\lambda}\left(F\left(x_{n}, y_{n}\right), g x\right) \cdot d^{\lambda}\left(F\left(y_{n}, x_{n}\right), g y\right)
\end{array}\right) \cdot d\left(g x_{n+1}, g x\right) \cdot d\left(g y_{n+1}, g y\right) \\
& =\phi\left(\begin{array}{c}
d^{\lambda}\left(g x, g x_{n}\right) \cdot d^{\lambda}\left(g y, g y_{n}\right), \\
d^{\lambda}(F(x, y), g x) \cdot d^{\lambda}(F(y, x), g y), \\
d^{\lambda}\left(g x_{n+1}, g x_{n}\right) \cdot d^{\lambda}\left(g y_{n+1}, g y_{n}\right), \\
d^{\lambda}\left(F(x, y), g x_{n}\right) \cdot d^{\lambda}\left(F(y, x), g y_{n}\right), \\
d^{\lambda}\left(g x_{n+1}, g x\right) \cdot d^{\lambda}\left(g y_{n+1}, g y\right)
\end{array}\right) \cdot d\left(g x_{n+1}, g x\right) \cdot d\left(g y_{n+1}, g y\right) .
\end{aligned}
$$

Let $\mathrm{n} \rightarrow \infty$ in the above inequality, we obtain

$$
\begin{aligned}
& d(F(x, y), g x) \cdot d(F(y, x), g y) \\
& \leqslant \phi\left(1, d^{\lambda}(F(x, y), g x) \cdot d^{\lambda}(F(y, x), g y), 1, d^{\lambda}(F(x, y), g x) \cdot d^{\lambda}(F(y, x), g y), 1\right) \cdot 1 \cdot 1 \\
& \leqslant \psi\left(d^{\lambda}(F(x, y), g x) \cdot d^{\lambda}(F(y, x), g y)\right) \\
& \leqslant d^{\lambda}(F(x, y), g x) \cdot d^{\lambda}(F(y, x), g y) .
\end{aligned}
$$

We have that $\lambda \in(0,1)$, such that $d(F(x, y), g x) \cdot d(F(y, x), g y)=1$, which implies that $d(F(x, y), g x)=1$ and $d(F(y, x), g y)=1$, where we have to prove that $F(x, y)=g x$ and $F(y, x)=g y$. Hence, $(g x, g y)$ is a coupled point of coincidence of mappings $F$ and $g$.

Now, we claim that a coupled point of coincidence is unique. Suppose that there is another $\left(x^{*}, y^{*}\right) \in$ $X \times X$ such $\left(g x^{*}, g y^{*}\right)$ is a coupled point of mappings $F$ and $g$, then by (2.1) we have

$$
\begin{aligned}
& d\left(g x, g x^{*}\right) \cdot d\left(g y, g y^{*}\right) \\
& =d\left(F(x, y), F\left(x^{*}, y^{*}\right)\right) \cdot d\left(F(y, x), F\left(y^{*}, x^{*}\right)\right) \\
& \leqslant \phi\left(\begin{array}{c}
d^{\lambda}\left(g x, g x^{*}\right) \cdot d^{\lambda}\left(g y, g y^{*}\right), \\
d^{\lambda}(F(x, y), g x) \cdot d^{\lambda}(F(y, x), g y), \\
d^{\lambda}\left(F\left(x^{*}, y^{*}\right), g x^{*}\right) \cdot d^{\lambda}\left(F\left(y^{*}, x^{*}\right), g y^{*}\right), \\
d^{\lambda}\left(F(x, y), g x^{*}\right) \cdot d^{\lambda}\left(F(y, x), g y^{*}\right), \\
d^{\lambda}\left(F\left(x^{*}, y^{*}\right), g x\right) \cdot d^{\lambda}\left(F\left(y^{*}, x^{*}\right), g x\right)
\end{array}\right) \\
& =\phi\left(\begin{array}{c}
d^{\lambda}\left(g x, g x^{*}\right) \cdot d^{\lambda}\left(g y, g y^{*}\right), \\
d^{\lambda}(g x, g x) \cdot d^{\lambda}(g y, g y), \\
d^{\lambda}\left(g x^{*}, g x^{*}\right) \cdot d^{\lambda}\left(g y^{*}, g y^{*}\right), \\
d^{\lambda}\left(g x, g x^{*}\right) \cdot d^{\lambda}\left(g y, g y^{*}\right), \\
d^{\lambda}\left(g x^{*}, g x\right) \cdot d^{\lambda}\left(g y^{*}, g y\right)
\end{array}\right) \\
& =\phi\left(d^{\lambda}\left(g x, g x^{*}\right) \cdot d^{\lambda}\left(g y, g y^{*}\right), 1,1, d^{\lambda}\left(g x, g x^{*}\right) \cdot d^{\lambda}\left(g y, g y^{*}\right), d^{\lambda}\left(g x^{*}, g x\right) \cdot d^{\lambda}\left(g y^{*}, g y\right)\right) \\
& \leqslant \psi\left(d^{\lambda}\left(g x, g x^{*}\right) \cdot d^{\lambda}\left(g y, g y^{*}\right)\right) \\
& \leqslant d^{\lambda}\left(g x, g x^{*}\right) \cdot d^{\lambda}\left(g y, g y^{*}\right) .
\end{aligned}
$$

From the above inequality, we must have $d\left(g x, g x^{*}\right) \cdot d\left(g y, g y^{*}\right)=1$, so that $d\left(g x, g x^{*}\right)=1$ and $d\left(g y, g y^{*}\right)=$ 1 , we obtain $g x=g x^{*}$ and $g y=g y^{*}$. Hence, $(g x, g y)$ is a unique coupled point of coincidence of mappings $\mathrm{F}$ and $\mathrm{g}$. 
Next we prove that $g x=g y$. In fact, from (2.1) we have

$$
\begin{aligned}
d(g x, g y) \cdot d(g y, g x) & =d(F(x, y), F(y, x)) \cdot d(F(y, x), F(x, y)) \\
& \leqslant \phi\left(\begin{array}{c}
d^{\lambda}(g x, g y) \cdot d^{\lambda}(g y, g x), \\
d^{\lambda}(F(x, y), g x) \cdot d^{\lambda}(F(y, x), g y), \\
d^{\lambda}(F(y, x), g y) \cdot d^{\lambda}(F(x, y), g x), \\
d^{\lambda}(F(x, y), g y) \cdot d^{\lambda}(F(y, x), g x), \\
d^{\lambda}(F(y, x), g x) \cdot d^{\lambda}(F(x, y), g y)
\end{array}\right) \\
& =\phi\left(d^{\lambda}(g x, g y) \cdot d^{\lambda}(g y, g x), 1,1, d^{\lambda}(g x, g y) \cdot d^{\lambda}(g y, g x), d^{\lambda}(g y, g x) \cdot d^{\lambda}(g x, g y)\right) \\
& \leqslant \psi\left(d^{\lambda}(g x, g y) \cdot d^{\lambda}(g y, g x)\right) \\
& \leqslant d^{\lambda}(g x, g y) \cdot d^{\lambda}(g y, g x) .
\end{aligned}
$$

We have $\lambda \in(0,1)$, so that $d(g x, g y) \cdot d(g y, g x)=1$, that means $d(g x, g y)=1, d(g y, g x)=1$, we obtain that $g x=g y$. Thus, $(g x, g x)$ is a unique coupled point of coincidence of mappings $F$ and $g$.

Now we show that $F$ and $g$ have a unique common coupled fixed point. For this, let $g x=u$. Then we have $u=g x=F(x, x)$. By the $\omega^{*}$-compatibility of $F$ and $g$, we have

$$
g u=g(g x)=g F(x, x)=F(g x, g x)=F(u, u) .
$$

Thus, $(g u, g u)$ is coupled point of coincidence of $F$ and $g$. By the uniqueness of a coupled point of coincidence, we have $g u=g x$. Thus, we obtain $u=g u=F(u, u)$. Therefore, $(u, u)$ is the unique common coupled fixed point of $\mathrm{F}$ and $\mathrm{g}$. This completes the proof of Theorem 2.1.

Remark 2.2. Theorem 2.1 generalizes and extends the coupled fixed point to multiplicative metric spaces, and so far nobody studied that, therefore it is very significative to do this.

Example 2.3. Let $X=[0,1]$, and $(X, d)$ be a multiplicative metric space defined by $d(x, y)=e^{|x-y|}$ for all $x, y \in X$. Let $F: X \times X \rightarrow X$ and $g: X \rightarrow X$ be two mappings defined by

$$
\mathrm{F}(\mathrm{x}, \mathrm{y})=\frac{x+y}{4}, \mathrm{gx}=10 \mathrm{x}
$$

for all $x, y \in X$. Now, for $(x, y),(u, v) \in X \times X$, we have

$$
\begin{aligned}
d(F(x, y), F(u, v)) \cdot d(F(y, x), F(v, u)) & =d\left(\frac{x+y}{4}, \frac{u+v}{4}\right) \cdot d\left(\frac{y+x}{4}, \frac{v+u}{4}\right) \\
& =e^{\left|\frac{x+y}{4}-\frac{u+v}{4}\right|} \cdot e^{\left|\frac{y+x}{4}-\frac{v+u}{4}\right|}=e^{\left|\frac{x+y}{2}-\frac{u+v}{2}\right|} .
\end{aligned}
$$

At the same time, $F, g$ satisfy the condition (2.1) with $\lambda=\frac{1}{2}$ and $\phi\left(t_{1}, t_{2}, t_{3}, t_{4}, t_{5}\right)=\left(t_{1} \cdot t_{2} \cdot t_{3} \cdot t_{4} \cdot t_{5}\right)^{\frac{1}{5}}$. In fact, we have

$$
\begin{gathered}
\left(\begin{array}{c}
\mathrm{d}^{\lambda}(g x, g u) \cdot d^{\lambda}(g y, g v), \\
d^{\lambda}(F(x, y), g x) \cdot d^{\lambda}(F(y, x), g y), \\
d^{\lambda}(F(u, v), g u) \cdot d^{\lambda}(F(v, u), g v), \\
d^{\lambda}(F(x, y), g u) \cdot d^{\lambda}(F(y, x), g v), \\
d^{\lambda}(F(u, v), g x) \cdot d^{\lambda}(F(v, u), g y)
\end{array}\right) \\
=\phi\left(\begin{array}{c}
d^{\lambda}(10 x, 10 u) \cdot d^{\lambda}(10 y, 10 v), \\
d^{\lambda}\left(\frac{x+y}{4}, 10 x\right) \cdot d^{\lambda}\left(\frac{y+x}{4}, 10 y\right), \\
d^{\lambda}\left(\frac{u+v}{4}, 10 u\right) \cdot d^{\lambda}\left(\frac{v+u}{4}, 10 v\right), \\
d^{\lambda}\left(\frac{x+y}{4}, 10 u\right) \cdot d^{\lambda}\left(\frac{y+x}{4}, 10 v\right), \\
d^{\lambda}\left(\frac{u+v}{4}, 10 x\right) \cdot d^{\lambda}\left(\frac{v+u}{4}, 10 y\right)
\end{array}\right)
\end{gathered}
$$




$$
\begin{aligned}
& \phi\left(\begin{array}{c}
e^{|10 x-10 y| \cdot \frac{1}{2}} \cdot e^{|10 y-10 v| \cdot \frac{1}{2}}, \\
e^{\left|\frac{x+y}{4}-10 x\right| \cdot \frac{1}{2}} \cdot e^{\left|\frac{y+x}{4}-10 y\right| \cdot \frac{1}{2}}, \\
e^{\left|\frac{u+v}{4}-10 u\right| \cdot \frac{1}{2}} \cdot e^{\left|\frac{v+u}{4}-10 v\right| \cdot \frac{1}{2}}, \\
e^{\left|\frac{x+y}{4}-10 u\right| \cdot \frac{1}{2}} \cdot e^{\left|\frac{\mid y+x}{4}-10 v\right| \cdot \frac{1}{2}}, \\
e^{\left|\frac{u+v}{4}-10 x\right| \cdot \frac{1}{2}} \cdot e^{\frac{\mid v+u}{4}-10 y \mid \cdot \frac{1}{2}}
\end{array}\right) \\
= & \left(\begin{array}{c}
e^{|5 x-5 u|+|5 y-5 v|}, \\
e^{\left|\frac{y}{8}-\frac{39 x}{8}\right|+\left|\frac{x}{8}-\frac{39 y}{8}\right|}, \\
e^{\left|\frac{v}{8}-\frac{39 u}{8}\right|+\left|\frac{u}{8}-\frac{39 v}{8}\right|,} \\
e^{\left|\frac{x+y}{8}-5 u\right|+\left|\frac{x+y}{8}-5 v\right|}, \\
e^{\left|\frac{u+v}{8}-5 x\right|+\left|\frac{u+v}{8}-5 y\right|}
\end{array}\right) \\
= & \left(e^{|5 x-5 u|+|5 y-5 v|+\left|\frac{y}{8}-\frac{39 x}{8}\right|+\left|\frac{x}{8}-\frac{39 y}{8}\right|+\left|\frac{v}{8}-\frac{39 u}{8}\right|+\left|\frac{u}{8}-\frac{39 v}{8}\right|+\left|\frac{x+y}{8}-5 u\right|+\left|\frac{x+y}{8}-5 v\right|+\left|\frac{u+v}{8}-5 x\right|+\left|\frac{u+v}{8}-5 y\right| \mid}\right)^{\frac{1}{5}} \\
\geqslant & \left(e^{\left|5 x-5 u+5 y-5 v+\frac{y}{8}-\frac{39 x}{8}+\frac{x}{8}-\frac{39 y}{8}+\frac{v}{8}-\frac{39 u}{8}+\frac{u}{8}-\frac{39 v}{8}+\frac{x+y}{8}-5 u+\frac{x+y}{8}-5 v+\frac{u+v}{8}-5 x+\frac{u+v}{8}-5 y\right|}\right){ }^{\frac{1}{5}} \\
= & \left(e^{\left|-\frac{9 x}{2}-\frac{9 y}{2}-\frac{29 u}{2}-\frac{29 v}{2}\right|}\right)^{\frac{1}{5}} \\
= & \left(e^{\left|\frac{9 x}{2}+\frac{9 y}{2}+\frac{29 u}{2}+\frac{29 v}{2}\right|}\right)^{\frac{1}{5}}=e^{\left|\frac{9 x}{10}+\frac{9 y}{10}+\frac{29 u}{10}+\frac{29 v}{10}\right|} \geqslant e^{\left|\frac{x+y}{2}-\frac{u+v}{2}\right|}=d(F(x, y), F(u, v)) \cdot d(F(y, x), F(v, u))
\end{aligned}
$$

for all $(x, y),(u, v) \in X \times X$. It is obvious that $F$ is $w^{*}$-compatible with $g$. Hence, all the conditions of Theorem 2.1 are satisfied. Moreover, $(0,0)$ is the unique common coupled fixed point of $F$ and $g$.

By taking $\phi\left(t_{1}, t_{2}, t_{3}, t_{4}, t_{5}\right)=\max \left\{t_{1}, t_{2}, t_{3}, t_{4}, t_{5}\right\}$ in Theorem 2.1, we have the following results.

Corollary 2.4. Let $(\mathrm{X}, \mathrm{d})$ be a multiplicative metric space, and $\mathrm{F}: \mathrm{X} \times \mathrm{X} \rightarrow \mathrm{X}$ and $\mathrm{g}: \mathrm{X} \rightarrow \mathrm{X}$ be two mappings. Suppose that there exists $\lambda \in(0,1)$ such that the condition

$$
d(F(x, y), F(u, v)) \cdot d(F(y, x), F(v, u)) \leqslant \max \left\{\begin{array}{c}
d^{\lambda}(g x, g u) \cdot d^{\lambda}(g y, g v), \\
d^{\lambda}(F(x, y), g x) \cdot d^{\lambda}(F(y, x), g y), \\
d^{\lambda}(F(u, v), g u) \cdot d^{\lambda}(F(v, u), g v), \\
d^{\lambda}(F(x, y), g u) \cdot d^{\lambda}(F(y, x), g v), \\
d^{\lambda}(F(u, v), g x) \cdot d^{\lambda}(F(v, u), g y)
\end{array}\right\}
$$

holds for all $(x, y),(u, v) \in X \times X$. If $F(X \times X) \subset g(X)$ and $g(X)$ is a multiplicative complete subspace of $X$, and $F$ and $\mathrm{g}$ are $\mathrm{\omega}^{*}$-compatible, then $\mathrm{F}$ and $\mathrm{g}$ have a unique common coupled fixed point of the form $(\mathrm{u}, \mathrm{u}) \in \mathrm{X} \times \mathrm{X}$.

Corollary 2.5. Let $(\mathrm{X}, \mathrm{d})$ be a multiplicative metric space, and $\mathrm{F}: \mathrm{X} \times \mathrm{X} \rightarrow \mathrm{X}$ and $\mathrm{g}: \mathrm{X} \rightarrow \mathrm{X}$ be two mappings. Suppose that there exists $\lambda \in(0,1)$ and $0<k \leqslant 1$ such that the condition

$$
d(F(x, y), F(u, v)) \cdot d(F(y, x), F(v, u)) \leqslant k \max \left\{\begin{array}{c}
d^{\lambda}(g x, g u) \cdot d^{\lambda}(g y, g v), \\
d^{\lambda}(F(x, y), g x) \cdot d^{\lambda}(F(y, x), g y), \\
d^{\lambda}(F(u, v), g u) \cdot d^{\lambda}(F(v, u), g v), \\
\frac{1}{2} d^{\lambda}(F(x, y), g u) \cdot d^{\lambda}(F(y, x), g v), \\
\frac{1}{2} d^{\lambda}(F(u, v), g x) \cdot d^{\lambda}(F(v, u), g y)
\end{array}\right\}
$$

holds for all $(x, y),(u, v) \in X \times X$. If $F(X \times X) \subset g(X)$ and $g(X)$ is a multiplicative complete subspace of $X$, and $\mathrm{F}$ and $\mathrm{g}$ are $\omega^{*}$-compatible, then $\mathrm{F}$ and $\mathrm{g}$ have a unique common coupled fixed point $(\mathrm{u}, \mathrm{u}) \in \mathrm{X} \times \mathrm{X}$, satisfying $\mathrm{u}=\mathrm{gu}=\mathrm{F}(\mathrm{u}, \mathrm{u})$.

Proof. Since (2.5) implies (2.4), so we can obtain Corollary 2.5.

Let $g=I_{X}$ (the identity mapping) in Theorem 2.1, and Corollaries 2.4 and 2.5. Then we have the following results. 
Corollary 2.6. Let $(\mathrm{X}, \mathrm{d})$ be a complete multiplicative metric space, and $\mathrm{F}: \mathrm{X} \times \mathrm{X} \rightarrow \mathrm{X}$ be a mapping. Suppose that there exists $\lambda \in(0,1)$ such that the condition

$$
d(F(x, y), F(u, v)) \cdot d(F(y, x), F(v, u)) \leqslant \phi\left(\begin{array}{c}
d^{\lambda}(x, u) \cdot d^{\lambda}(y, v), \\
d^{\lambda}(F(x, y), x) \cdot d^{\lambda}(F(y, x), y), \\
d^{\lambda}(F(u, v), u) \cdot d^{\lambda}(F(v, u), v), \\
d^{\lambda}(F(x, y), u) \cdot d^{\lambda}(F(y, x), v), \\
d^{\lambda}(F(u, v), x) \cdot d^{\lambda}(F(v, u), y)
\end{array}\right)
$$

holds for all $(x, y),(u, v) \in X \times X$. Then $F$ has a unique coupled fixed point of the form $(u, u) \in X \times X$, which satisfies $\mathrm{u}=\mathrm{F}(\mathrm{u}, \mathrm{u})$.

Corollary 2.7. Let $(\mathrm{X}, \mathrm{d})$ be a complete multiplicative metric space, and $\mathrm{F}: \mathrm{X} \times \mathrm{X} \rightarrow \mathrm{X}$ be a mapping. Suppose that there exists $\lambda \in(0,1)$ such that the condition

$$
d(F(x, y), F(u, v)) \cdot d(F(y, x), F(v, u)) \leqslant \max \left\{\begin{array}{c}
d^{\lambda}(x, u) \cdot d^{\lambda}(y, v), \\
d^{\lambda}(F(x, y), x) \cdot d^{\lambda}(F(y, x), y), \\
d^{\lambda}(F(u, v), u) \cdot d^{\lambda}(F(v, u), v), \\
d^{\lambda}(F(x, y), u) \cdot d^{\lambda}(F(y, x), v), \\
d^{\lambda}(F(u, v), x) \cdot d^{\lambda}(F(v, u), y)
\end{array}\right\}
$$

holds for all $(\mathrm{x}, \mathrm{y}),(\mathrm{u}, v) \in \mathrm{X} \times \mathrm{X}$. Then $\mathrm{F}$ has a unique coupled fixed point of the form $(\mathrm{u}, \mathrm{u}) \in \mathrm{X} \times \mathrm{X}$, which satisfies $\mathrm{u}=\mathrm{F}(\mathrm{u}, \mathrm{u})$.

Corollary 2.8. Let $(\mathrm{X}, \mathrm{d})$ be a complete multiplicative metric space, and $\mathrm{F}: \mathrm{X} \times \mathrm{X} \rightarrow \mathrm{X}$ be a mapping. Suppose that there exists $\lambda \in(0,1)$ and $0<k \leqslant 1$ satisfying

$$
d(F(x, y), F(u, v)) \cdot d(F(y, x), F(v, u)) \leqslant k \max \left\{\begin{array}{c}
d^{\lambda}(x, u) \cdot d^{\lambda}(y, v), \\
d^{\lambda}(F(x, y), x) \cdot d^{\lambda}(F(y, x), y), \\
d^{\lambda}(F(u, v), u) \cdot d^{\lambda}(F(v, u), v), \\
\frac{1}{2} d^{\lambda}(F(x, y), u) \cdot d^{\lambda}(F(y, x), v), \\
\frac{1}{2} d^{\lambda}(F(u, v), x) \cdot d^{\lambda}(F(v, u), y)
\end{array}\right\}
$$

for all $(x, y),(u, v) \in X \times X$. Then $F$ has a unique coupled fixed point $(u, u) \in X \times X$, satisfying $u=F(u, u)$.

Theorem 2.9. Let $(\mathrm{X}, \mathrm{d})$ be a multiplicative metric space, and $\mathrm{F}: \mathrm{X} \times \mathrm{X} \rightarrow \mathrm{X}$ and $\mathrm{g}: \mathrm{X} \rightarrow \mathrm{X}$ be two mappings. Suppose that there exists $\lambda \in(0,1)$ such that

$$
\begin{aligned}
& d(F(x, y), F(u, v)) \cdot d(F(y, x), F(v, u)) \\
& \leqslant a_{1} d^{\lambda}(g x, g u) \cdot d^{\lambda}(g y, g v)+a_{2} d^{\lambda}(F(x, y), g x) \cdot d^{\lambda}(F(y, x), g y) \\
& \quad+a_{3} d^{\lambda}(F(u, v), g u) \cdot d^{\lambda}(F(v, u), g v)+a_{4} d^{\lambda}(F(x, y), g u) \cdot d^{\lambda}(F(y, x), g v) \\
& \quad+a_{5} d^{\lambda}(F(u, v), g x) \cdot d^{\lambda}(F(v, u), g y)
\end{aligned}
$$

holds for all $(x, y),(u, v) \in X \times X$, where $a_{i} \geqslant 0(i=1,2, \cdots, 5)$ with

$$
0<a_{1}+a_{2}+a_{3}+a_{4}+a_{5} \leqslant 1 .
$$

If $\mathrm{F}(\mathrm{X} \times \mathrm{X}) \subset \mathrm{g}(\mathrm{X})$ and $\mathrm{g}(\mathrm{X})$ is a multiplicative complete subspace of $\mathrm{X}$, then $\mathrm{F}$ and $\mathrm{g}$ have a unique common coupled point of coincidence $(\mathrm{gx}, \mathrm{gy}) \in \mathrm{X} \times \mathrm{X}$, satisfying $\mathrm{gx}=\mathrm{F}(\mathrm{x}, \mathrm{y})=\mathrm{gy}=\mathrm{F}(\mathrm{y}, \mathrm{x})$.

Moreover, if $\mathrm{F}$ and $\mathrm{g}$ are $\omega^{*}$-compatible, then $\mathrm{F}$ and $\mathrm{g}$ have a unique common coupled fixed point $(\mathrm{u}, \mathrm{u}) \in \mathrm{X} \times \mathrm{X}$, satisfying $\mathrm{u}=\mathrm{gu}=\mathrm{F}(\mathrm{u}, \mathrm{u})$. 
Proof. For all $x, y, u, v \in X$, let

$$
M(x, y, u, v) \leqslant \max \left\{\begin{array}{c}
d^{\lambda}(g x, g u) \cdot d^{\lambda}(g y, g v), \\
d^{\lambda}(F(x, y), g x) \cdot d^{\lambda}(F(y, x), g y), \\
d^{\lambda}(F(u, v), g u) \cdot d^{\lambda}(F(v, u), g v), \\
d^{\lambda}(F(x, y), g u) \cdot d^{\lambda}(F(y, x), g v), \\
d^{\lambda}(F(u, v), g x) \cdot d^{\lambda}(F(v, u), g y)
\end{array}\right\} .
$$

Then from (2.6) we have

$$
\begin{aligned}
& a_{1} d^{\lambda}(g x, g u) \cdot d^{\lambda}(g y, g v)+a_{2} d^{\lambda}(F(x, y), g x) \cdot d^{\lambda}(F(y, x), g y)+a_{3} d^{\lambda}(F(u, v), g u) \cdot d^{\lambda}(F(v, u), g v) \\
& \left.\quad+a_{4} d^{\lambda}(F(x, y), g u) \cdot d^{\lambda}(F(y, x), g v)+a_{5} d^{\lambda}(F(u, v), g x) \cdot d^{\lambda}(F(v, u), g y)\right) \\
& \leqslant\left(a_{1}+a_{2}+a_{3}+a_{4}+a_{5}\right) M(x, y, u, v) \leqslant M(x, y, u, v) .
\end{aligned}
$$

Therefore, the result of Theorem 2.9 can be obtained from Corollary 2.4 immediately.

In Theorem 2.9, if we take $\alpha=a_{1}$ and $a_{2}=a_{3}=a_{4}=a_{5}=0$, we deduce the following corollary.

Corollary 2.10. Let $(X, d)$ be a complete multiplicative metric space, and suppose that there exists $\lambda \in(0,1)$ and let $\mathrm{F}: \mathrm{X} \times \mathrm{X} \rightarrow \mathrm{X}$ be a mapping satisfying

$$
d(F(x, y), F(u, v)) \cdot d(F(y, x), F(v, u)) \leqslant \alpha d^{\lambda}(g x, g u) \cdot d^{\lambda}(g y, g v)
$$

for all $(x, y),(\mathrm{u}, v) \in \mathrm{X} \times \mathrm{X}$, where $0<\alpha \leqslant 1$. If $\mathrm{F}(\mathrm{X} \times \mathrm{X}) \subset \mathrm{g}(\mathrm{X})$ and $\mathrm{g}(\mathrm{X})$ is a multiplicative complete subspace of $\mathrm{X}$, then $\mathrm{F}$ and $\mathrm{g}$ have a unique common coupled point of coincidence $(\mathrm{gx}, \mathrm{gy}) \in \mathrm{X} \times \mathrm{X}$, satisfying $g x=F(x, y)=g y=F(y, x)$.

Moreover, if $\mathrm{F}$ and $\mathrm{g}$ are $\mathrm{\omega}^{*}$-compatible, then $\mathrm{F}$ and $\mathrm{g}$ have a unique common coupled fixed point $(\mathrm{u}, \mathrm{u}) \in \mathrm{X} \times \mathrm{X}$, satisfying $\mathrm{u}=\mathrm{gu}=\mathrm{F}(\mathrm{u}, \mathrm{u})$.

In Theorem 2.9, if we take $\alpha=a_{1}, \beta=a_{4}$ and $a_{2}=a_{3}=a_{5}=0$, we deduce the following result.

Corollary 2.11. Let $(\mathrm{X}, \mathrm{d})$ be a complete multiplicative metric space, and suppose that there exists $\lambda \in(0,1)$ and let $\mathrm{F}: \mathrm{X} \times \mathrm{X} \rightarrow \mathrm{X}$ be a mapping satisfying

$$
d(F(x, y), F(u, v)) \cdot d(F(y, x), F(v, u)) \leqslant \alpha d^{\lambda}(g x, g u) \cdot d^{\lambda}(g y, g v)+\beta d^{\lambda}(F(x, y), g u) \cdot d^{\lambda}(F(y, x), g v) .
$$

for all $(x, y),(u, v) \in X \times X$, where $0<\alpha+\beta \leqslant 1$. If $F(X \times X) \subset g(X)$ and $g(X)$ is a multiplicative complete subspace of $\mathrm{X}$, then $\mathrm{F}$ and $\mathrm{g}$ have a unique common coupled point of coincidence $(\mathrm{gx}, \mathrm{gy}) \in \mathrm{X} \times \mathrm{X}$, satisfying $g x=F(x, y)=g y=F(y, x)$.

Moreover, if $\mathrm{F}$ and $\mathrm{g}$ are $\omega^{*}$-compatible, then $\mathrm{F}$ and $\mathrm{g}$ have a unique common coupled fixed point $(\mathrm{u}, \mathrm{u}) \in \mathrm{X} \times \mathrm{X}$, satisfying $\mathrm{u}=\mathrm{gu}=\mathrm{F}(\mathrm{u}, \mathrm{u})$.

In Theorem 2.9, taking $\alpha=a_{1}, \beta=a_{2}, \gamma=a_{4}$ and $a_{3}=a_{5}=0$, we deduce the following result.

Corollary 2.12. Let $(X, d)$ be a complete multiplicative metric space, and suppose that there exists $\lambda \in(0,1)$ and let $\mathrm{F}: \mathrm{X} \times \mathrm{X} \rightarrow \mathrm{X}$ be a mapping satisfying

$$
\begin{aligned}
d(F(x, y), F(u, v)) \cdot d(F(y, x), F(v, u)) \leqslant & \alpha d^{\lambda}(g x, g u) \cdot d^{\lambda}(g y, g v)+\beta d^{\lambda}(F(x, y), g x) \cdot d^{\lambda}(F(y, x), g y) \\
& +\gamma d^{\lambda}(F(x, y), g u) \cdot d^{\lambda}(F(y, x), g v)
\end{aligned}
$$

for all $(x, y),(u, v) \in X \times X$, where $0<\alpha+\beta+\gamma \leqslant 1$. If $F(X \times X) \subset g(X)$ and $g(X)$ is a multiplicative complete subspace of $\mathrm{X}$, then $\mathrm{F}$ and $\mathrm{g}$ have a unique common coupled point of coincidence (gx,gy) $\in \mathrm{X} \times \mathrm{X}$, satisfying $g x=F(x, y)=g y=F(y, x)$. 
Moreover, if $\mathrm{F}$ and $\mathrm{g}$ are $\omega^{*}$-compatible, then $\mathrm{F}$ and $\mathrm{g}$ have a unique common coupled fixed point $(u, u) \in X \times X$, satisfying $u=g u=F(u, u)$.

Let $\mathrm{g}=\mathrm{I}_{\mathrm{X}}$ (the identity mapping) in Theorem 2.9, and Corollaries 2.10, 2.11, and 2.12. Then we have the following results.

Corollary 2.13. Let $(\mathrm{X}, \mathrm{d})$ be a complete multiplicative metric space, and $\mathrm{F}: \mathrm{X} \times \mathrm{X} \rightarrow \mathrm{X}$ be a mapping. Suppose that there exists $\lambda \in(0,1)$ such that

$$
\begin{aligned}
& d(F(x, y), F(u, v)) \cdot d(F(y, x), F(v, u)) \\
& \leqslant \\
& \quad a_{1} d^{\lambda}(x, u) \cdot d^{\lambda}(y, v)+a_{2} d^{\lambda}(F(x, y), x) \cdot d^{\lambda}(F(y, x), y) \\
& \quad+a_{3} d^{\lambda}(F(u, v), u) \cdot d^{\lambda}(F(v, u), v)+a_{4} d^{\lambda}(F(x, y), u) \cdot d^{\lambda}(F(y, x), v) \\
& \quad+a_{5} d^{\lambda}(F(u, v), x) \cdot d^{\lambda}(F(v, u), y)
\end{aligned}
$$

holds for all $(x, y),(u, v) \in X \times X$, where $a_{i} \geqslant 0(i=1,2, \cdots, 5)$ with

$$
0<a_{1}+a_{2}+a_{3}+a_{4}+a_{5} \leqslant 1 .
$$

Then $\mathrm{F}$ has a unique coupled fixed point $(\mathrm{u}, \mathrm{u}) \in \mathrm{X} \times \mathrm{X}$, satisfying $\mathrm{u}=\mathrm{gu}=\mathrm{F}(\mathrm{u}, \mathrm{u})$.

Corollary 2.14. Let $(\mathrm{X}, \mathrm{d})$ be a complete multiplicative metric space, and $\mathrm{F}: \mathrm{X} \times \mathrm{X} \rightarrow \mathrm{X}$ be a mapping. Suppose that there exists $\lambda \in(0,1)$ such that

$$
d(F(x, y), F(u, v)) \cdot d(F(y, x), F(v, u)) \leqslant \alpha d^{\lambda}(x, u) \cdot d^{\lambda}(y, v)+\beta d^{\lambda}(F(x, y), u) \cdot d^{\lambda}(F(y, x), v) .
$$

for all $(x, y),(u, v) \in X \times X$, where $0<\alpha+\beta \leqslant 1$. Then $F$ has a unique coupled fixed point of the form $(\mathrm{u}, \mathrm{u}) \in \mathrm{X} \times \mathrm{X}$, satisfying $\mathrm{u}=\mathrm{F}(\mathrm{u}, \mathrm{u})$.

Corollary 2.15. Let $(\mathrm{X}, \mathrm{d})$ be a complete multiplicative metric space, and $\mathrm{F}: \mathrm{X} \times \mathrm{X} \rightarrow \mathrm{X}$ be a mapping. Suppose that there exists $\lambda \in(0,1)$ such that

$$
\begin{aligned}
& d(F(x, y), F(u, v)) \cdot d(F(y, x), F(v, u)) \\
& \leqslant \alpha d^{\lambda}(x, u) \cdot d^{\lambda}(y, v)+\beta d^{\lambda}(F(x, y), x) \cdot d^{\lambda}(F(y, x), y)+\gamma d^{\lambda}(F(x, y), u) \cdot d^{\lambda}(F(y, x), v)
\end{aligned}
$$

for all $(x, y),(u, v) \in X \times X$, where $0<\alpha+\beta+\gamma \leqslant 1$. Then $F$ has a unique coupled fixed point of the form $(\mathrm{u}, \mathrm{u}) \in \mathrm{X} \times \mathrm{X}$, satisfying $\mathrm{u}=\mathrm{F}(\mathrm{u}, \mathrm{u})$.

Example 2.16. Let $X=[0,1]$, and $(X, d)$ be a multiplicative metric space defined by

$$
d(x, y)=e^{|x-y|}
$$

for all $x, y$ in $X$. Let $\lambda=\frac{1}{4}$ and let $F: X \times X \rightarrow X, g: X \rightarrow X$ as

$$
F(x, y)=\frac{1}{4} \ln (1+|x-y|), g x=2 x, \forall x, y \in X .
$$

By the definition, we can easily obtain that $F(X \times X) \subseteq g X$. Next we show that the pair $(F, g)$ is $\omega^{*}$ compatible. In fact

$$
\left\{\begin{array} { l } 
{ F ( x , y ) = g x , } \\
{ F ( y , x ) = g y . }
\end{array} \Leftrightarrow \left\{\begin{array}{l}
\frac{1}{4} \ln (1+|x-y|)=2 x, \\
\frac{1}{4} \ln (1+|y-x|)=2 y .
\end{array} \Leftrightarrow x=y=0 .\right.\right.
$$

This shows that $(g 0, g 0)$ is the unique coupled point of coincidence point of mappings $F$ and $g$. Apparently, we get $F(g 0, g 0)=g(F(0,0))=0$, therefore the pair $(F, g)$ is $\omega^{*}$-compatible. 
In the following, we will prove that the condition (2.7) holds in Corollary 2.10 with $\alpha=1$. In fact, $\forall(x, y),(u, v) \in X \times X$, we have

$$
\begin{aligned}
& d(F(x, y), F(u, v)) \cdot d(F(y, x), F(v, u)) \\
& =e^{\left|\frac{1}{4} \ln (1+|x-y|)-\frac{1}{4} \ln (1+|u-v|)\right|} \cdot e^{\left|\frac{1}{4} \ln (1+|y-x|)-\frac{1}{4} \ln (1+|v-u|)\right|} \\
& =e^{\left|\frac{1}{4} \ln \frac{1+|x-y|}{1+|u-v|}\right|} \cdot e^{\left|\frac{1}{4} \ln \frac{1+|y-x|}{1+|v-u|}\right|} \\
& =e^{\left|\frac{1}{4} \ln \frac{1+|u-v|+|x-y|-|u-v|}{1+|u-v|}\right|} \cdot e^{\left|\frac{1}{4} \ln \frac{1+|v-u|+|y-x|-|v-u|}{1+|v-u|}\right|} \\
& =e^{\left|\frac{1}{4} \ln \left(1+\frac{|x-y|-|u-v|}{1+|u-v|}\right)\right|} \cdot e^{\left|\frac{1}{4} \ln \left(1+\frac{|y-x|-|v-u|}{1+|v-u|}\right)\right|} \\
& =e^{\left|\frac{1}{2} \ln \left(1+\frac{|x-y|-|u-v|}{1+|u-v|}\right)\right|} \\
& \leqslant e^{\left|\frac{1}{2} \ln \left(1+\frac{|(x-y)-(u-v)|}{1+|u-v|}\right)\right|} \\
& =e^{\left|\frac{1}{2} \ln \left(1+\frac{|(x-\mathfrak{u})-(y-v)|}{1+|u-v|}\right)\right|} \\
& \leqslant e^{\left|\frac{1}{2} \ln \left(1+\frac{|x-u|+|y-v|}{1+|u-v|}\right)\right|} \\
& \leqslant e^{\left|\frac{1}{2} \ln [1+|x-u|+|y-v|]\right|} \\
& \leqslant e^{\left|\frac{1}{2}(|x-u|+|y-v|)\right|}=\left(e^{2|x-u|} \cdot e^{2|y-v|}\right)^{\frac{1}{4}} \\
& =d^{\frac{1}{4}}(g x, g u) \cdot d^{\frac{1}{4}}(g y, g v) \leqslant 1 \cdot d^{\frac{1}{4}}(g x, g u) \cdot d^{\frac{1}{4}}(g y, g v) .
\end{aligned}
$$

Then the mappings $\mathrm{F}$ and $\mathrm{g}$ satisfy all the conditions appearing in Corollary 2.10, by the result of Corollary 2.10 , we get $F$ and $g$ have a unique coupled common fixed point. In fact, $(0,0)$ is a unique coupled common fixed point of $F$ and $g$, that is $F(0,0)=g 0=0$.

\section{Application to integral equations}

In this section, we wish to study the existence and uniqueness problem of solution for a class of nonlinear integral equations by using the obtained result.

Throughout this section, we assume that $X=C[0,1]$ is the set of all continuous functions defined on $[0,1]$. Define $d: X \times X \rightarrow \mathbb{R}^{+}$by

$$
d(x, y)=\sup _{p \in[0,1]} e^{|x(p)-y(p)|}, \forall x, y \in X
$$

Then we get $(X, d)$ is multiplicative metric space.

Consider the following nonlinear integral equations

$$
\left\{\begin{array}{l}
x(p)=h(p)+\int_{0}^{1} k(p, q)\left(f_{1}(q, x(q))+f_{2}(q, y(q))\right) d q, \quad p \in[0,1], \\
y(p)=h(p)+\int_{0}^{1} k(p, q)\left(f_{1}(q, y(q))+f_{2}(q, x(q))\right) d q, \quad p \in[0,1], \quad \forall x, y \in X,
\end{array}\right.
$$

where the functions $h:[0,1] \rightarrow \mathbb{R}, k:[0,1] \times[0,1] \rightarrow \mathbb{R}^{+}$, and $f_{1}, f_{2}:[0,1] \times \mathbb{R} \rightarrow \mathbb{R}$.

Next, we will analyze (3.1) under the following conditions:

(i) $h, k, f_{1}$, and $f_{2}$ are continuous functions;

(ii) there exist constants $\sigma, \tau>0$ such that

$$
\left\{\begin{array}{l}
\left|f_{1}(p, x)-f_{1}(p, y)\right| \leqslant \sigma|x-y| \\
\left|f_{2}(p, x)-f_{2}(p, y)\right| \leqslant \tau|x-y|
\end{array}\right.
$$

$\forall p \in[0,1], x, y \in \mathbb{R}$ 
(iii) $0<\lambda=2 \max \{\sigma, \tau\}\|k\|_{\infty} \leqslant 1$, where $\|k\|_{\infty}=\sup \{k(p, q): p, q \in[0,1]\}$.

Theorem 3.1. Under the conditions (i)-(iii), integral equation (3.1) has a unique common solution in $\mathrm{C}[0,1]$.

Proof. First, the operators $\mathrm{F}: \mathrm{X} \times \mathrm{X} \rightarrow \mathrm{X}$ and $\mathrm{g}: \mathrm{X} \rightarrow \mathrm{X}$ are defined respectively by

$$
F(x, y)(p)=h(p)+\int_{0}^{1} k(p, q)\left(f_{1}(q, x(q))+f_{2}(q, y(q))\right) d q, \quad p \in[0,1], \forall x, y, z \in X,
$$

and

$$
g x(p)=x(p), \forall x \in X .
$$

Then we induced $F(X \times X) \subset g(X), F$ and $g$ are $w^{*}$-compatible, and $g(X)$ is a complete subspace of $(X, d)$. From the definition of $S_{1}$, we can get

$$
\begin{aligned}
& d(F(x, y), F(u, v)) \cdot d(F(y, x), F(v, u)) \\
& =\sup _{p \in[0,1]} e^{|F(x, y)(p)-F(u, v)(p)|} \cdot \sup _{p \in[0,1]} e^{|F(y, x)(p)-F(v, u)(p)|} \\
& =\sup _{\mathbf{p} \in[0,1]} e^{\left|\left[h(p)+\int_{0}^{1} k(p, q)\left(f_{1}(\mathbf{q}, x(\mathbf{q}))+f_{2}(\mathbf{q}, y(q))\right) d q\right]-\left[h(p)+\int_{0}^{1} k(p, q)\left(f_{1}(\mathbf{q}, \mathfrak{u}(\mathbf{q}))+f_{2}(\mathbf{q}, v(\mathbf{q}))\right) d \mathbf{q}\right]\right|}
\end{aligned}
$$

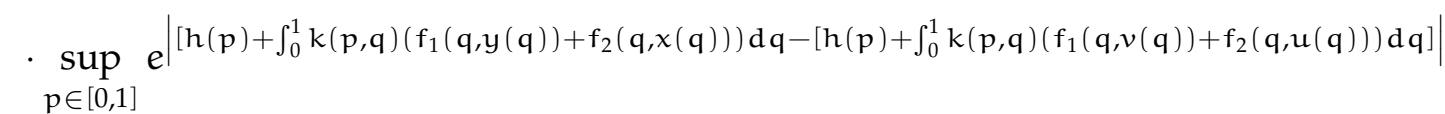

$$
\begin{aligned}
& =\sup _{\mathbf{p} \in[0,1]} e^{\left|\int_{0}^{1} k(p, q)\left(f_{1}(\mathbf{q}, x(\mathbf{q}))+f_{2}(\mathbf{q}, y(q))\right) d q-\int_{0}^{1} k(p, q)\left(f_{1}(\mathbf{q}, \mathbf{u}(\mathbf{q}))+f_{2}(\mathbf{q}, v(\mathbf{q}))\right) d q\right|} \\
& \cdot \sup e^{\left|\int_{0}^{1} k(p, q)\left(f_{1}(q, y(q))+f_{2}(q, x(q))\right) d q-\int_{0}^{1} k(p, q)\left(f_{1}(q, v(q))+f_{2}(q, u(q))\right) d q\right|} \\
& p \in[0,1] \\
& =\sup _{\mathbf{p} \in[0,1]} e^{\left|\int_{0}^{1} k(p, q)\left[\left(f_{1}(\mathbf{q}, x(\mathbf{q}))-f_{1}(\mathbf{q}, \mathbf{u}(\mathbf{q}))\right)+\left(f_{2}(\mathbf{q}, \mathbf{y}(\mathbf{q}))-f_{2}(\mathbf{q}, v(\mathbf{q}))\right)\right]\right| d q} \\
& \text { - } \sup e^{\mid \int_{0}^{1} k(p, q)\left[\left(f_{1}(q, y(q))-f_{1}(q, v(q))\right)+\left(f_{2}(q, x(q))-f_{2}(q, u(q))\right)\right] d q} \\
& p \in[0,1] \\
& \leqslant \sup e^{\int_{0}^{1} k(p, q)\left[\left|f_{1}(q, x(q))-f_{1}(q, u(q))\right|+\left|f_{2}(q, y(q))-f_{2}(q, v(q))\right|\right] d q} \\
& p \in[0,1] \\
& \text {. } \sup _{\mathbf{p} \in[0,1]} e^{\int_{0}^{1} k(p, q)\left[\left|f_{1}(q, y(q))-f_{1}(q, v(q))\right|+\left|f_{2}(q, x(q))-f_{2}(q, u(q))\right|\right] d q} .
\end{aligned}
$$

By the condition (ii), we get

$$
\left\{\begin{array}{l}
\left|f_{1}(p, x(q))-f_{1}(p, y(q))\right| \leqslant \sigma|x(q)-y(q)| \\
\left|f_{2}(p, x(q))-f_{2}(p, y(q))\right| \leqslant \tau|x(q)-y(q)|
\end{array}\right.
$$

Then inequality (3.2) becomes

$$
\begin{aligned}
& d(F(x, y), F(u, v)) \cdot d(F(y, x), F(v, u)) \leqslant \sup _{p \in[0,1]} e^{\int_{0}^{1} k(p, q)(\sigma|x(q)-u(q)|+\tau|y(q)-v(q)|) d q} \\
& +\sup _{\mathbf{p} \in[0,1]} e^{\int_{0}^{1} k(p, q)(\sigma|y(q)-v(q)|+\tau|x(q)-u(q)|) d q} \\
& \leqslant\left(\sup _{\mathbf{p} \in[0,1]} e^{\int_{0}^{1} k(p, q)(|x(q)-u(q)|+|y(q)-v(q)|) d q}\right)^{\max \{\sigma, \tau\}} \\
& \cdot\left(\sup _{\mathbf{p} \in[0,1]} e^{\int_{0}^{1} k(p, q)(|y(q)-v(q)|+|x(q)-u(q)|) d q}\right)^{\max \{\sigma, \tau\}} .
\end{aligned}
$$


By using Cauchy-Schwartz inequality, we have

$$
\begin{aligned}
e^{\int_{0}^{1} k(p, q)(|x(\mathbf{q})-u(q)|+|y(q)-v(q)|) d q} & \leqslant e^{\left(\int_{0}^{1} k^{2}(\mathbf{p}, \mathbf{q}) d \mathbf{q}\right)^{\frac{1}{2}}\left(\int_{0}^{1}(|x(\mathbf{q})-\mathfrak{u}(\mathbf{q})|+|\mathbf{y}(\mathbf{q})-v(\mathbf{q})|)^{2} \mathbf{d q}\right)^{\frac{1}{2}}} \\
& \leqslant\left(e^{\sup _{\mathbf{p} \in[0,1]}|x(\mathbf{q})-\mathfrak{u}(\mathbf{q})|+\sup _{\mathbf{p} \in[0,1]}|\mathbf{y}(\mathbf{q})-v(\mathbf{q})|}\right)^{\|k\|_{\infty}} .
\end{aligned}
$$

Similarly, we can prove that

$$
\begin{aligned}
e^{\int_{0}^{1} k(p, q)(|y(q)-v(q)|+|x(q)-u(q)|) d q} & \leqslant e^{\left(\int_{0}^{1} k^{2}(\mathbf{p}, \mathbf{q}) d \mathbf{q}\right)^{\frac{1}{2}}\left(\int_{0}^{1}(|y(q)-v(\mathbf{q})|+|x(\mathbf{q})-\mathfrak{u}(\mathbf{q})|)^{2} \mathbf{d} \mathbf{q}\right)^{\frac{1}{2}}} \\
& \leqslant\left(e^{\sup _{\mathbf{p} \in[0,1]}|\mathbf{y}(\mathbf{q})-v(\mathbf{q})|+\sup _{\mathbf{p} \in[0,1]}|x(\mathbf{q})-\mathfrak{u}(\mathbf{q})|}\right)^{\|k\|_{\infty}} .
\end{aligned}
$$

Substituting (3.4) and (3.5) into (3.3), we obtain

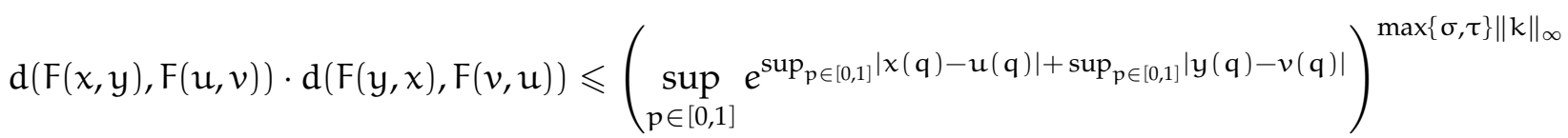

$$
\begin{aligned}
& \cdot\left(\sup _{\mathbf{p} \in[0,1]} e^{\sup _{\mathbf{p} \in[0,1]}|y(q)-v(q)|+\sup _{\mathbf{p} \in[0,1]}|x(\mathbf{q})-\mathfrak{u}(\mathbf{q})|}\right)^{\max \{\sigma, \tau\}\|k\|_{\infty}} \\
& =\left(\sup _{\mathbf{p} \in[0,1]} e^{|x(\mathbf{q})-\mathfrak{u}(\mathbf{q})|+|\boldsymbol{y}(\mathbf{q})-v(\mathbf{q})|}\right)^{\max \{\sigma, \tau\}|| k \|_{\infty}} \\
& \cdot\left(\sup _{\mathbf{p} \in[0,1]} e^{|\boldsymbol{y}(\mathbf{q})-v(\mathbf{q})|+|x(\mathbf{q})-\mathfrak{u}(\mathbf{q})|}\right)^{\max \{\sigma, \tau\}\|k\|_{\infty}} \\
& \leqslant\left(\sup _{p \in[0,1]} e^{|x(\mathbf{q})-u(q)|} \cdot \sup _{\mathbf{p} \in[0,1]} e^{|\boldsymbol{y}(\mathbf{q})-v(\mathbf{q})|}\right)^{2 \max \{\sigma, \tau\}\|k\|_{\infty}} \\
& =\left(\sup _{p \in[0,1]} e^{|g x(q)-g u(q)|} \cdot \sup _{p \in[0,1]} e^{|g y(q)-g v(q)|}\right)^{2 \max \{\sigma, \tau\}\|k\|_{\infty}} \\
& =d^{2 \max \{\sigma, \tau\}\|k\|_{\infty}}(g x, g u) \cdot d^{2 \max \{\sigma, \tau\}\|k\|_{\infty}}(g y, g v) \text {. }
\end{aligned}
$$

It is easily seen that the condition (2.7) holds with $\alpha=1$. Then it is obvious that $F$ and $g$ satisfy all the conditions appearing in Corollary 2.10, consequently, it follows from the result of Corollary 2.10 that $F$ and $g$ have a unique common coupled fixed point $(u, u)$, satisfying $F(u, u)=g u=u$. So $(u, u)$ is the unique solution of integral equation (3.1).

Example 3.2. Consider the following functional integral equation set:

$$
\left\{\begin{array}{l}
x(p)=\frac{p}{1+\sqrt{p}}+\int_{0}^{1} \frac{\sin (q \cdot \pi)}{8+p} \cdot\left[\frac{e^{-p x(q)}}{9}+\frac{\sin p}{10} \cdot \frac{|y|}{1+|y(q)|}\right] d q \\
y(p)=\frac{p}{1+\sqrt{p}}+\int_{0}^{1} \frac{\sin (q \cdot \pi)}{8+p} \cdot\left[\frac{e^{-p y(q)}}{9}+\frac{\sin p}{10} \cdot \frac{|x|}{1+|x(q)|}\right] d q
\end{array}\right.
$$

where the operators $h:[0,1] \rightarrow \mathbb{R}, k:[0,1] \times[0,1] \rightarrow \mathbb{R}^{+}$, and $f_{1}, f_{2}:[0,1] \times \mathbb{R} \rightarrow \mathbb{R}$, are defined respectively by

$$
h(p)=\frac{p}{1+\sqrt{p}}, k(p, q)=\frac{\sin (q \cdot \pi)}{8+p}, f_{1}(p, x)=\frac{e^{-p x}}{9}, f_{2}(p, x)=\frac{\sin p}{10} \cdot \frac{|x|}{1+|x|} .
$$


It is easily seen that $h(p), k(p, q), f_{1}(p, x), f_{2}(p, x)$ are continuous functions. Next, since

$$
\begin{aligned}
\left|f_{1}(p, x)-f_{1}(p, y)\right| & =\left|\frac{e^{-p x}}{9}-\frac{e^{-p y}}{9}\right|=\left|\frac{-p \cdot e^{-p \xi}}{9}(x-y)\right| \leqslant \frac{1}{9}|x-y| \\
\left|f_{2}(p, x)-f_{2}(p, y)\right| & =\left|\frac{\sin p}{10} \cdot \frac{|x|}{1+|x|}-\frac{\sin p}{10} \cdot \frac{|y|}{1+|y|}\right| \\
& \leqslant \frac{1}{10}\left|\frac{|x|}{1+|x|}-\frac{|y|}{1+|y|}\right| \\
& =\frac{1}{10}\left|\left(1-\frac{1}{1+|x|}\right)-\left(1-\frac{1}{1+|y|}\right)\right| \\
& =\frac{1}{10}\left|\frac{1}{1+|y|}-\frac{1}{1+|x|}\right| \\
& =\frac{1}{10}\left|-\frac{1}{(1+\varepsilon)^{2}}(|x|-|y|)\right| \\
& \leqslant \frac{1}{10}|| x|-| y|| \leqslant \frac{1}{10}|x-y|
\end{aligned}
$$

where $p \in[0,1], \xi$ exists between $x$ and $y$, and $\varepsilon$ exists between $|x|$ and $|y|$. Then we have $\sigma=\frac{1}{9}, \tau=\frac{1}{10}$, $\|k\|_{\infty}=\sup \{k(p, q): p, q \in[0,1]\}=\frac{1}{8}$, thus

$$
2 \max \{\sigma, \tau\}\|k\|_{\infty}=\frac{1}{36}<1 .
$$

Consequently, all the conditions of Theorem 3.1 are satisfied. Hence the integral equation set (3.6) has a unique solution in $\mathrm{C}[0,1]$.

\section{Acknowledgment}

This work is supported by the National Natural Science Foundation of China $(11071169,11271105)$, the Natural Science Foundation of Zhejiang Province (Y6110287).

\section{References}

[1] M. Abbas, B. Ali, Y. I. Suleiman, Common fixed points of locally contractive mappings in multiplicative metric spaces with application, Int. J. Math. Math. Sci., 2015 (2015), 7 pages. 1

[2] M. Abbas, M. Ali Khan, S. Redenović, Common coupled fixed point theorems in cone metric spaces for w-compatible mappings, Appl. Math. Comput., 217 (2010), 195-202. 1, 1.13

[3] M. Abbas, A. R. Khan, T. Nazir, Coupled common fixed point results in two generalized metric spaces, Appl. Math. Comput., 217 (2011), 6328-6336. 1

[4] A. E. Bashirov, E. M. Kurpınar, A. Özyapıc1, Multiplicative calculus and its applications, J. Math. Anal. Appl., 337 (2008), 36-48. 1, 1.1, 1.2, 1.3

[5] A. E. Bashirov, E. Misırlı, Y. Tandoğdu, A. Özyapıcı, On modeling with multiplicative differential equations, Appl. Math. J. Chinese Univ. Ser. B, 26 (2012), 425-438. 1

[6] Y. J. Cho, M. H. Shah, N. Hussain, Coupled fixed points of weakly F-contractive mappings in topological spaces, Appl. Math. Lett., 24 (2011), 1185-1190. 1

[7] T. Gnana Bhaskar, V. Lakshmikantham, Fixed point theorems in partially ordered metric spaces and applications, Nonlinear Anal., 65 (2006), 1379-1393. 1, 1.11

[8] F. Gu, Some new common coupled fixed point results in two generalized metric spaces, Fixed point Theory Appl., 2013 (2013), 21 pages. 1

[9] F. Gu. Y. J. Cho, Common fixed point results for four maps satisfying $\phi$-contractive condition in multiplicative metric spaces, Fixed Point Theory Appl., 2015 (2015), 19 pages. 1

[10] F. Gu, L. Wang, Some coupled fixed-point theorems in two quasi-partial metric spaces, Fixed point Theory Appl., 2015 (2015), 17 pages. 1 
[11] F. Gu, Y. Yin, A new common coupled fixed point theorem in generalized metric space and applications to integral equations, Fixed Point Theory Appl., 2013 (2013), 17 pages.

[12] F. Gu, S.-H. Zhou, Coupled common fixed point theorems for a pair of commuting mappings in partially ordered G-metric spaces, Fixed Point Theory Appl., 2013 (2013), 18 pages. 1

[13] X.-J. He, M.-M. Song, D.-P. Chen, Common fixed points for weak commutative mappings on a multiplicative metric space, Fixed Point Theory Appl., 2013 (2013), 9 pages. 1

[14] E. Karapınar, Couple fixed point theorems for nonlinear contractions in cone metric spaces, Comput. Math. Appl., 59 (2010), 3656-3668. 1

[15] V. Lakshmikantham, Lj. Ćirić, Coupled fixed point theorems for nonlinear contractions in partially ordered metric spaces, Nonlinear Anal., 70 (2009), 4341-4349. 1, 1.12

[16] M. Özavşar, A. C. Çevikel, Fixed points of multiplicative contraction mappings on multiplicative metric spaces, ArXiv, 2012 (2012), 14 pages. 1, 1.4, 1.5, 1.6, 1.7, 1.8, 1.9, 1.10

[17] B. Samet, Coupled fixed point theorems for a generalized Meir-Keeler contraction in partially ordered metric spaces, Nonlinear Anal., 72 (2010), 4508-4517. 1

[18] W. Shatanawi, On w-compatible mappings and common coupled coincidence point in cone metric spaces, Appl. Math. Lett., 25 (2012), 925-931.

[19] W. Shatanawi, B. Samet, M. Abbas, Coupled fixed point theorems for mixed monotone mappings in ordered partial metric spaces, Math. Comput. Modelling, 55 (2012), 680-687. 1 